\title{
The Future E-Living for Elderly: The Use of Remote Controlled Devices
}

\author{
$\underline{\text { doi:10.3991/ijoe.v6i1.1179 }}$ \\ S. Lam ${ }^{1}$ and W. Chung ${ }^{2}$ \\ ${ }^{1}$ Oxford University, UK \\ ${ }^{2}$ Hospital Authority, Hong Kong
}

\begin{abstract}
Elderly populations are growing globally, the need for assistive technologies to facilitate ageing-in-place becomes more prominent for researchers and legislators. Understanding the use of remote controlled devices is crucial to optimize design and application strategies that may reduce caregiver burden, extend healthy ageing in place, and minimize demands on the health care system. This paper will establish strategies for assistance to the elderly in ageing-in-place for the future e-living environment. This research study is to explore attitudes, opinions, and preferences of older adults concerning the use of online technology to support and extend their ability to "ageing in place." Four major themes emerged as important for older adults to age in place: safety and independence, social interaction, use of technology in the past, and the desire for support.
\end{abstract}

Index Terms-E-living, Remote controlled devices, Elderly housing, Smart home

\section{INTRODUCTION}

In today's ageing society, ageing-in-place in combination with a sufficient amount of professional home care is commonly promoted as a strategy for maintaining autonomy, independence, sense of identity, and well-being. The wish to remain living independently, regardless of the condition of housing, neighbourhood, and health, is often a personal choice of older adults themselves [1], but is influenced by someone's health status or that of a partner, as well as the ability of the partner to cope with the burden of care posed upon him or her. On the individual level, this desire leads to home modifications, moving, or simply living under less favourable conditions. Older adults do not comprise a homogenous population, particularly in terms of lifestyle and health status. Health problems vary in severity and mix, and tend to increase with ageing.

\section{A. The changing landscape of ageing demographics}

Ageing is the social factor shaping the future of the vast majority of nation's today. A combination of longer life and decreased fertility rates are driving a demographic transition from the once 'normal' population distribution of many young people with few older people to a distribution that reflects many more people over age 50 than there are children. Industrialized economies, primarily Organization for Economic Cooperation and Development or OECD states will face accelerated ageing when the baby boomers of North America and Europe and Dankai of Japan begin turning 65. Europe, already with more older people than children, will have twice the number of elderly than children by 2050. Even developing economies, such as China, are now facing a virtual geriatric explosion with nearly 140 million people already over 60 . In fact, the United Nations revised World Population Prospects estimates that by 2045 humankind will have set a historical first where the number of people over age 60 will be greater than the number of children under age 15 .

\section{B. New demand of an ageing society}

The number of elderly individuals requiring services will increase dramatically over the next two decades. Individuals are living longer and wish to remain in their homes as they age. Older adults almost uniformly prefer to age in place [2-3]. The nature of long-term care is changing as well. Throughout the $1990 \mathrm{~s}$ and into the $21^{\mathrm{st}}$ century, different models of combining health and housing services to facilitate ageing in place have developed. The private market is beginning to discover that the high-end assisted living model may not be as sustainable as previously thought. The approach to ageing services is beginning to shift. People are more aware of the changing nature of the country's population - fewer younger, taxpaying workers and more retirees on fixed incomes. A few states have taken note of the impending demographic shift and have started long-term planning efforts to prepare for the growth in their elderly population, focusing on the use of technology and more cost-efficient options for delivering care. The assistance the elderly need is provided most often by informal family caregivers [4] who frequently experience significant stress while juggling multiple roles in addition to their caregiving duties [5]. In this ageing society, there is evidence that technological advancements could help older adults (especially those living alone) to live independently and alleviate the pressures on their caregivers [6-7].

\section{Ageing Services Technologies}

Several studies [8-10] have investigated the interest to live in a home with IT technology. Other researchers [7, 11] argue that technologies such as activity monitoring devices and advanced communication interfaces can both reduce caregiver work load and reduce demands on the health care system. For example, remote monitoring technology allows caregivers to track an older person's activity. Networks of environmental sensors can determine location and movement and can monitor sleep patterns. A significant body of literature highlights the importance of the home environment in supporting competencies and quality of life for people as they age. Reference [12] conducted a comprehensive review of literature in ageing and environment that showcased Lawton's Ecological Theory of Ageing-Competency-Environment Model. 
Only equal to the rate of ageing has been the rapid development of advanced technology. The creative application of new technology to healthcare has received considerable attention from researchers and investment from both business and government. Biomedical, diagnostic technologies and other applied areas are greatly improving the lives of older people. Other technologies are being developed to improve health as part of daily living in the home, at work or in some instance, even the car. Robotics, for example, is offering the possibility of 'carebots' to assist older people with activities of daily living from dressing, cleaning the home, to reminding them to take medication [13].

Remote online devices are perhaps the richest area to produce new ideas to support the health of older adults. Remote online devices are being used to monitor, manage and motivate a new generation of healthcare for older people [14]. For example, sensors, related algorithms and attending call centers form the basis of 'smart home health' technologies providing families and formal caregivers with the capacity to remotely monitor the wellbeing of an older adult living athome [15]. Pervasive computing applications are available to predict a fall based upon a change in gait, rather than simply report a disaster after the often fatal event has occurred. Telemedicine or telecare is increasingly common providing connectivity with clinicians to improve the management of chronic disease in the comfort of an older person's home rather than in the costly surroundings of a hospital [16-17]. Other devices from the ubiquitous cell phone to intelligent everyday devices such cabinets, picture frames, kitchen appliances and toilets are being used to motivate people to adhere to diets, take medication or to exercise.

\section{Case study}

Japan and Hong Kong are both rapidly ageing societies in Asia, where adoption of new technology happens quite fast. Japan is advanced in service robotics research and even commercialization of home robots. Co-operation in this home service field of robotics will provide information of future use of robots in homes and in helping the senior citizens. People will also profit from the purely technical information that can be retrieved from cooperation with Japanese universities and companies.

Senior housing in the US is a well developed business even though the US is not a rapidly ageing society like many European countries and Japan. However there are a great number of American senior citizens, who are living in special senior housing compounds. The US does not have the problem of finding people to do the daily service jobs for the seniors. However, home automation in the US is most developed in the world and that and other technologies are used to improve the quality of service for seniors. The US is a leading "service industry" nation. Thus advanced combinations of technology and service can be found there.

Germany is the biggest economy in Europe with a growing number of elderly people. We can learn how the Germans are solving the problems of how to serve the senior citizens in a good way without spending too much money on it. Germany is also an attractive market area for Finnish "well-being products"
1) U.S.

In U.S, American Baptist Homes of the West (ABHOW), headquartered East of San Francisco, has embraced high-tech to deliver high-touch services [18]. ABHOW leadership has recognized that technology can be vital to seniors' well-being, particularly when new tools facilitate communication, lifelong learning and residents' ability to continue living independently. ABHOW is currently working to standardize the nurse call systems, which now differ from one community to the next. The call systems let a resident who needs help send an alert to first responders by pushing a button on a pendant worn around the neck or wrist. The system informs staff if there has been a lack of a normal range of daily activity by the apartment residents, who may not interact as frequently with care staff as other residents. Door sensors provide supplemental activity data for residents who have pets that could trigger motion sensors. ABHOW emphasizes that residents' privacy will be preserved and that the sensors are an optional service enhancement. At Alzheimer's care centers, anti-wandering and external video systems are being installed to improve resident safety.

\section{2) Hong Kong}

In Hong Kong, The Senior Citizen Home Safety Association (SCHSA) was founded by a group of Hong Kong citizens in 1996 after an incident during a cold spell in which more than 100 elderly citizens living alone were found dead. To prevent a recurrence of the tragedy, SCHSA was formed and now provides a number of services to the elderly in Hong Kong with the aim of enhancing their quality of life through the development and promotion of various services.

The organization's mission is: to provide a 24-hour emergency assistant service to the elderly; to respond to both the psychological and physiological needs of the elderly through the hotline service; to mobilize the community by establishing a volunteer network that will enhance the well-being of the elderly and create a harmonious living environment for them; and to fill the current service gaps and to supplement the existing services for the elderly.

The SCHSA mainly provides the Personal Emergency Link (PE Link) service and the Elder Ring Hotline service to Hong Kong's elderly. Currently, SCHSA is the only non-profit charitable organisation that offers a 24-hour PE Link emergency support service for millions of elderly citizens living alone as well as for other people in need. SCHSA perhaps is the most successful organization in the region applying modern online remote technology in elderly services.

SCHSA also launched the Mobile Link service on 30 November 2008. It is an innovative outdoor support and caring service, which provides 24-hour support service to elderly people aged 60 or above. With the use of a specially designed, one-button Mobile Link device and utilizing GSM mobile technology, users can easily seek assistance from the SCHSA's 24-hour call centre by simply pressing the button or pulling a strap. In case the users are lost, their approximate locations and whereabouts can be tracked to allow authorities to locate them. This innovative device allows the elderly to enjoy an active lifestyle in an outdoor environment. 


\section{3) Germany}

The Future Senior Living project is cooperating with Diakonisches Werk - Innere Mission- im Kirchenkreis Minden e.V. on the subject of Assistive Automation for independent living. The Minden Deaconesses' Institution takes care of home services for the elderly and disabled in their area in Northern Germany. They are also developing new technology for this purpose. More over they have manufactured facilities for producing systems and devices that can be used to improve safety and quality of living at home. Discussions with the Minden Deaconesses' Institution are in progress. The contact person is the Chairman of the Board of the Institution pastor Frank Stefan.

\section{AIM \& OBJECTIVE}

The aim of this paper is generation of a conceptual model / strategy that incorporates technology as a compensatory mechanism in the person environment interaction to facilitate e-living environment for elderly. The model is discussed in a following section. This model is primarily based on integration of the key concepts from the current literature. However, the authors are conducting a series of studies with older adults living in their own homes and in assisted living facilities. The preliminary data analysis of our first focus group session revealed useful concepts for the strategy generation. We highlight some of these emerging themes from that session.

Research questions that guided these pilot projects were as follows:

- How do older adults perceive use of technology?

- What factors influence older adults' perceptions about use of communication and monitoring technology?

\section{METHOD}

The authors investigated attitudes, opinions, and preferences of technologies that have the potential to enhance older adults' safety, health, social interaction, and sense of independence with a group of older adults living in elderly public estates in Hong Kong.

\section{A. Subjects}

Data were collected with a small sample of nine cognitively intact older adult residents. Special designed focus group interviews were conducted. Participants were asked their views about the use of remote online technologies to support $e$-living. Nine in-home monitoring systems were assembled and installed in the homes of clients of a home health care agency managed in Hong Kong. The total sample size was 9 participants, five males, and eight females. All participants were over the age of 65. All subjects were local Chinese. Inclusion criteria required the subjects to be ambulatory, be able to provide for their own hygiene except for bathing (some participants received assistance from home health aides in bathing), be able to prepare meals and eat autonomously, be eligible for home health and have a friend or relative who acted as an informal caregiver who was also willing to participate in the study. Exclusion criteria included subject refusal to being monitored, inability to get out of bed, the requirement for extensive outside assistance in the activities of daily living, and not having an informal caregiver who was willing to participate.

\section{B. Measurement}

To begin, the authors asked participants to think about the circumstances that led to their decision to enter assisted living. Questions that guided the subsequent discussion were as follows: What issues and concerns do you think you encounter in living independently? What kinds of assistance were most needed? What do you think about these (communication and monitoring) devices (demonstrated during the scenario presentation)? Would you like to have access to this kind of technology? Who would you talk to with this device (communication technology)? Who should have access to such information (your movements in the screen; monitoring technology)? What problems can you see with this kind of technology?

Four major themes emerged from the focus group interviews related to remote online technology issues: safety (number of mention $[n]=54)$, social interactions $(n=25)$, use of technology $(n=22)$ and support $(n=15)$. Three additional themes-health, finance, and privacy-though not directly linked to technology by the participants, were also identified as important to them. In this paper, we discuss the top four themes: safety, social interaction, use of technology, and support.

\section{RESULTS}

Safety, especially related to falling, was the main concern of the participants. Most participants seemed to have a fear of falling and were concerned about how they could get help if they fall. Throughout this discussion, participants often mentioned their experiences about falling; they described accidents in which their friends or neighbors fell down and received help from professionals.

Social interaction is another central theme for the participants. Interacting with other people is crucial for their well-being. For example, one participant mentioned the importance of social interactions in the assisted living facility, while another emphasized that the residents consider themselves a family.

Support is another theme that participants frequently brought up. This theme is also related to issues for safety, falling, and independence. When they talked about getting help, they always mentioned their experiences of falling. Receiving support is very important to the participants, especially when they fall. Furthermore, it seems that they would prefer to receive help from professionals rather than family members.

Social interaction - The participants might have developed a new way of family life in the facility, receive emotional support from their community. They want to be independent and enjoy interacting and supporting each other in the facility. The responses indicated that the participants prefer professionals to watch their movements using a monitoring technology, rather than burdening their family members. This may be related to the fact that they had already moved away from their family and saw the facility staff as their primary caregivers.

The participants were mainly concerned about safety and independence. In order to be safe and independent, they acknowledged the usefulness of technological devices and the importance of social interactions. Participants expressed their wish to age safely and successfully in the community. They were aware of potential uses for technology to help achieve this goal. They believed that technology is useful and helpful for them, and would al- 
low them to live safely and independently. The relationships among themes imply that older adults have primarily positive attitudes toward use of technological devices as shown in the diagram below, and they believe that advanced technology will help them live safely and independently and achieve their goal of optimal ageing. The authors have incorporated the themes of independence and safety in our conceptual model. Additionally, participants' strong motivations to be safe and independent significantly influenced their perceptions and attitudes toward use of advanced technology.

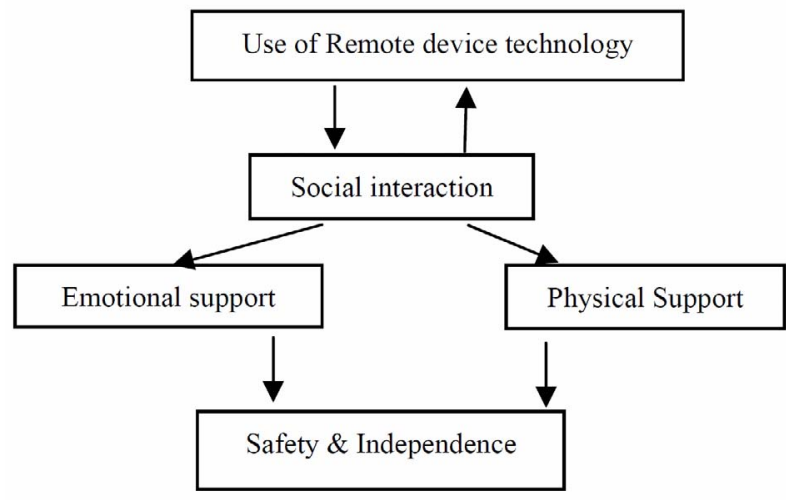

Figure 1. Themes and relationships from our study

\begin{abstract}
Using communication technological devices helps older adults to contact people in order to receive physical support. Receiving immediate physical support leads to safety. Social interactions in the residential community help older adults to have emotional support. Providing emotional support to each other in the community leads to a feeling of safety. There could be a relationship between use of technology and social interaction because use of technology may leads to social interactions or verse. The reason for it is that the better the social interaction, the more encouragement and support from their peer groups and hence the better use of technology.
\end{abstract}

In addition to the acceptance issue, the lack of clear incentives seems to be one of the biggest barriers to widespread implementation of online remote devices for elderly home, in particular, and also for online care although to a somewhat lesser extent. Economic and financial incentives are the big aspect of this, but quality (of service and outcome) incentives also need to be considered. This issue of incentives arises both at the level of individual providers themselves (hospitals, clinics, doctors, etc.) and also in relation to the distribution of the costs and benefits more generally for particular components of the overall system (social care, housing, healthcare; primary care versus hospitals; and so on). It also arises for payers, those who fund or otherwise reimburse the services that are provided.

Although online remote applications and related technologies have been available for many years, major corporations and governments have only recently given significant attention to the promise of technology to improve the lives of older adults. Recent interest, along with the profound change in demographics, suggests that 'technology and ageing' is moving toward the tipping point where interest will translate into investment to move countless inventions that exist in the laboratory into innovations in living. However, availability of technology and obvious need may not translate into commercial opportunity or policy success.

Usability remains an important issue to adoption according to our finding. While improvement have been made in design the user friendliness of devices remains a challenge. In addition to somewhat obvious characteristics, such as font size, controls for arthritic hands, research suggests that more work needs to be conducted to address the most effective mental model to guide the user's expectation of how the device may work. Related to usability and practical use is the perceived 'hassle factor.' The related logistics associated with installation, correct use, maintenance, etc., may be just great enough to outweigh the possible benefits of inviting new technology into an older person's daily routine.

Perhaps the most potent factor affecting the success or failure of technology adoption by older adults addresses how user's feel about what adopting the technology may say about them. For example, does adoption of a deviceenabled service to monitor my wellbeing and medication adherence trample my privacy and independence in the name of safety and security. Is technology assisting me or replacing my own sense of self-control and confidence?

While all the participants were intrigued by the potential of smart home technologies to support health and long-term independence, they did have some perceptions that may explain relatively slow adoption of many of these devices and related services. First, was the thought that many of the technologies offered considerable promise in improving personal safety but at considerable cost - not to income but to independence. A closely related perception was that smart technologies in the home may be designed for the oldest and not younger or more healthy older adults seeking to simply age-in-place. This perception may be a significant barrier to technology adoption because as the group admitted few adults of any age see themselves as 'old' and even fewer see themselves as frail. Consequently, some of the participants thought that smart technologies may be a "stigma to some older people” and more symbolic of their frailty than of their supported independence and health.

According to the interview results, the authors have summarized six components of chronic care management that are facilitated by the online remote technologies: (1) early intervention-to detect deterioration and intervene before unscheduled and preventable services are needed; (2) integration of care- - exchange of data and communication across multiple co-morbidities, multiple providers, and complex disease states; (3) coaching-motivational interviewing and other techniques to encourage patient behavioral change and self- care; (4) increased trustpatients' satisfaction and feelings of "connectedness" with providers; (5) workforce changes-shifts to lower-cost and more plentiful health care workers, including medical assistants, community health workers, and social workers; and (6) increased productivity-decreased home visit travel time and automated documentation.

\section{DISCUSSION}

\section{A. Barriers and Opportunities}

Several barriers to the advancement of ageing services technology have come into focus from the above result findings. First, advances in technology and their value 
proposition to care services are still largely unfamiliar to consumers, care providers and government officials. Continued education about technologies for each of these stakeholders will be necessary to expand awareness and spawn new innovation and partnership for government programs and private services. Furthermore, large-scale pilots are needed to generate quantitative data on the value and effectiveness of technology-enabled services.

Second, significant technical uncertainty about technology remains a barrier for organizations, including management and care staff, as well as for government program staff. Technical uncertainties include reliability and maintenance, interoperability, long-term viability, changing the interpersonal interview dynamic to accommodate technology, and user compliance. Technology companies will need to continue to address these concerns, provide superior customer support and education for users and providers, and design their products to be interoperable with other devices and allow effective exchange of standardized health information.

Third, demonstration of sustainable business models to support ageing service technologies in various care settings is still in its infancy. Furthermore, reimbursement from insurers and government programs to use technologies is nearly nonexistent. However, through the course of education and data collection, as well as finding common objectives with payers (as discussed below), we believe increasing levels of reimbursement is likely in the future despite fiscal constraints. Moreover, private ageing service providers are in a unique position to test and demonstrate sustainable business models with their private-pay consumers.

\section{B. Identifying Opportunities:}

The authors would like to suggest the followings as opportunities for various different players in the market.

\section{1) Elderly service providers}

Providers should pursue resources to plan and execute additional outcome-oriented field pilots and larger-scale demonstration projects. This is paramount in showing how technology can improve quality of care, consumer satisfaction and well being, staff efficiency, etc., with various populations of older adults in different care settings. Such findings can lead the way for government and other users to change traditional reimbursement policies and program structures to achieve these mutual goals.

Early adopters should raise awareness among policy makers about "best practices" of how providers have made strategic investments in technology to improve care as well as what barriers they face to achieve sustained business models and broad deployment of ageing service technologies. Critical to success is the need to address organizational integration and adoption of new work-flow strategies, development of innovative business models to sustain operations, and the provision of technical support personnel to manage new processes. Private ageing service providers who are primarily funded by private-pay consumers are uniquely positioned to modify operating practices and service delivery because they are not wholly dependent upon government.

\section{2) Technology Companies}

Technology companies must be called to action in order to enhance the technical capabilities of their technologies to realize the technology-enabled care vision. Important steps include achieving interconnectivity between different and disparate clinical information technology systems, which is needed to guarantee completeness and continuity of information between the home and long-term care settings and assuring continuity of care. Technology companies may wish to craft a common standard of practice to provide adequate technical support to ensure that emerging tools are dependable for consumers and care providers for on-going service operations. Companies should address acceptance and usability of technology by end-users. This might be achieved by taking a systematic approach to research and development that involves the participation of seniors, caregivers and providers in the products' design and development cycle.

\section{3) Government and Private Payers}

Government and private payers must be called to action to capitalize on the opportunity of technology-enabled care. Given their economies of scale, these payers have a unique ability to apply existing resources currently channeled through "legacy" reimbursement methods into an improved model of preventive care. Private payers are a critical participant in this call for action. Because of their ability to tailor a package of services to meet the needs of consumers within a certain amount of reimbursement (capitated payment), they are uniquely positioned to change traditional service and payment models. Health plans should seek partnerships with ageing service providers and technology companies to quantify the value of technology-enabled care and demonstrate economic and non-economic benefits for their plan's benefit populations. Because the care needs of the plans' beneficiaries are often comparable to those of public fee-for-service program consumers, quantitative findings will be especially informative for government payers and may potentially be drivers of change to reimbursement policies.

\section{SOME INITIAL POLICY IMPLiCATIONS}

This study is perhaps the first effort to develop a systematic and coherent baseline and mapping of the market situation for remote technology device for older people in Hong Kong. One important contribution is the identification of what seem to be the most promising / important opportunities at present, and this helps sort the 'hype' that one often finds with regard to technological possibilities from the reality of what can be expected to be achieved in the near- to mid-term. In this regard, the most coherent 'markets' for attention seem to be the online care market to support social care and independent living and the home online remote health market to support chronic disease management and home hospital services. Other areas for attention include the smart housing sectors and the wider assistive technologies sector, but both are currently very fragmented.

In regard to online care roll-out, in particular, some areas do not yet have the basic pre-requisite of a social alarm infrastructure in place. Social alarms have proved a useful component of home care and, in practice they often represent the baseline infrastructure (technical and organisational) upon which more sophisticated solutions are introduced. This is an aspect that could be targeted for infrastructural investment at national level. More generally, in some areas the necessary equipment and service supply chains for online care is not well developed. This is an area where innovative approaches to public-private 
partnerships can play an important role, with the public sector providing the main eventual 'market' but the private sector having a key role to play in supplying the necessary technology and also, in some cases, for out-sourcing various aspects of service provision as well.

Although infrastructural issues are important, the evidence suggests that various issues around acceptance of online care and online health programs within the mainstream social care and healthcare systems are amongst the biggest factors limiting market development to date.

\section{A. Awareness and appreciation of the (potential) value}

One dimension of this concerns lack of awareness and appreciation of the (potential) value of the online remote types of service innovation at policy, service provider and practitioner levels. The evidence suggests that many public funding bodies, and private households, are faced with the challenge to provide efficient care with limited financial resources. Applications that go beyond 1st generation are often considered as 'high cost' applications for which the business case is not immediately self evident. This is something that could be addressed both at Central government level and also at the local NGOs level through support for information exchange and other accompanying measures to develop and disseminate the necessary knowledge-base. As regards the knowledge base aspect, there is a need both to consolidate existing knowledge on the 'business' case and the 'value' case, and also to support the development of new knowledge. For this, support for large-scale trials seems to be an essential measure if sufficient real-life experience and knowledge is to be gained. There would also be value in dedicated cost-benefit research to provide a more solid evidence base and one that was better tuned to the varying situations across different segemnts. This would be helpful in supporting the achievement of a shared view of the business and quality benefits that can be achieved, as well as ensuring a realistic perspective that avoids reliance on the type of 'hype' sometimes associated with technological innovations.

\section{B. Skills and capacity for organisational change and innovation}

The evidence shows that challenges posed by lack of skills and capacity for organisational change and innovation to take advantage of new service concepts like online care and online health can also be important barriers. Beyond mere technological innovation, introduction of ICTenabled solutions into day to day care practices tends to require a considerable degree of organisational innovation. Apart from a need to overcome staff resistance to organisational change more generally, this may require training in the new skills and responsibilities required at the various stages of ICT-enabled service delivery (e.g. initial needs assessment, installation, maintenance and so on). More generally, online care solutions may often involve a need for multi-disciplinary approaches and may even require the emergence of new occupational profiles. An initial overview suggests that a general policy shift towards integrated care provision seems to have taken place in many countries. However, organisational and managerial difficulties have been reported to act as barriers towards achieving integrated services provision in practice. Some countries have given specific attention to these aspects and support for exchange of experiences and capac- ity building should therefore also be part of the policy approach.

\section{More formal medico-regulatory enablement}

More formal aspects of medico-legal enablement of online remote services also need policy attention as these can pose barriers to exploitation of the potential. As regards online care, an initial overview of the situation across the providers suggests that there may not be especially strong legal/regulatory barriers in most cases, especially as regards social alarms and also for second generation online care program. However, in some areas concerns about privacy/surveillance have been raised in relation to passive sensors and legislation is in place to regulate their use. It can be expected that such issues will come more strongly to the fore when further generation involving continuous activity monitoring and data processing become more visible in policy and practice. Establishment of a forum for exchange of experiences and perspectives across the different stakeholders, and across the government would be useful in this regard.

As regards online health program, there are issues of professional acceptability / encouragement to be considered. Already there are considerable differences across government as regards regulation and practice in relation to Internet communication consultation and electronic consultations, and some of these may also come to arise in relation to home online health. There is also the wider issue of the extent to which self-management of health is encouraged or discouraged, and how this may impinge on the market for home healthcare devices and services. Again, establishment of a forum for exchange of experiences this regard. Finally, there is also a cross-border dimension that may emerge as important as home online health services become more mainstream. This is linked to the more general cross-border mobility of patients seeking treatment that is now possible for local citizens, and the quite large numbers who avail of this. A realistic scenario, therefore, would be for a patient to be receiving home online health program services in their local area from a healthcare provider in their specific local hospitals. Apart from the social/healthcare issues outlined above, there are also the more general regulatory issues associated with public procurement in both online care and online health programs. As mentioned already, there is considerable potential for innovative utilisation of public procurement to stimulate the necessary supply and demand side coevolution, but this seems little used in practice so far. Government supported efforts to raise awareness and exchange experiences in this area would thus seem to be useful.

\section{CONCLUSION}

Advances in smart home technologies offer considerable power and promise to improve the health and wellness of older adults ageing-in-place and help to caregivers supporting frail elderly. All participants embraced the further development and commercialization of these technologies. However, a variety of concerns including usability, reliability, privacy and affordability were identified as possible barriers to adoption. In addition, while age is a critical factor, additional research should be done to understand how the use of ICT and related technology enabled services are impacted by the wide range of socioeconomic and cultural factors that exist in today's and 
tomorrow's older population. These findings validate previous research on technology adoption by older adults. However, this exploratory work reveals a new area worthy of further examination - how might institutional innovation occur to move inventions in the laboratory into the living rooms of older adults? Future research should also examine what creative financing and technology-enabled services might be offered through trusted providers, e.g., affinity groups, neighborhood associations, local government, banks, utilities, retail pharmacies, or insurers.

\section{A. More innovation designs are needed}

As outlined above, our preliminary findings shed light on a number of barriers to the mainstreaming of ICT enabled solutions that are in principle available today. They also indicate a continuing need for further technological innovation. This concerns both the development of new/improved systems and devices in general and the coevolution of technology and services in particular.

\section{B. Research \& Development $(R \& D)$}

Many of the basic technologies and system components for online remote devices are already available today, and the main market barriers relate to issues of acceptance and incentives. Nevertheless there is still a lot of $R \& D$ needed to improve existing products and develop new ones.

Some important areas for attention emerge from the preliminary evidence base as follows:

- Mobile devices and monitoring services

- End-user devices

- Monitoring and processing systems for service centres, including upgrades to address new technologies and new capabilities

- Clinical support systems to present relevant information and guidance to health and social care professionals

- Improved interoperability between the various system components and the different products and services

\section{From e-living to Ubiquitous?}

Demonstration "e-living” homes highlight technologies that really do enhance the quality of life for seniors by allowing them to live safely at home. It is also still a relatively new field with rapidly evolving technology. It is likely online remote controlled elderly services and activities of daily living (ADL) technologies will one day be integrated into a single system. This will help modify behavior so older adults can manage their health and ADL challenges more effectively. The authors believe that technology will become more passive, less intrusive, and more user-friendly to the customer. As technology continues to improve, more data will be available that can be used to enhance a person's daily living activities

Online remote patient monitoring is currently growing rapidly, bringing convenience and simplifying care for patients and health care professionals. In the future, not only is online remote patient monitoring expected to expand in terms of adoption, but also in terms of the variety of applications and offerings. The authors believe that an increase in the use of general broadband technology and the "wiredness" of homes, hospitals, and other care settings will facilitate the growth.
Home care agencies may well prove to be the most effective entities in the adoption of online remote technology. Unlike provider-based plans and home health agencies, the business model for hospital-based delivery systems has historically been poorly aligned with chronic care innovations and the online remote technologies that support them. After some early experimentation with online remote technologies, many health plans have relied upon disease management (DM) contractors to identify opportunities to use online remote technology in chronic care management.

Online remote technologies represent an opportunity to grapple with the coverage issues that arise when a category of technology is continuously and rapidly evolving. Whether it is by DM companies, health plans or homecare providers and senior living communities, coverage and reimbursement policies remain a barrier to remote technology management deployment. We have a great deal to learn about the most effective means of compensating providers for their use.

The prevalence of this technology will increase and the skills of providers will be tested not only by the quality of the patient-provider interaction, but by technological proficiency and access. In turn, medical facilities will encounter new challenges in preparing and training adequate personnel. The cost structures of medical facilities will change as capital investments in medicine will have to increase to respond to growing demand. Given its enormous potential, remote patient monitoring will continue its rapid growth, playing a large role in the future of medicine and treatment.

\section{REFERENCES}

[1] L.N. Gitlin. Next steps in home modification and assistive technology research. In N Charness, KW Schaie, editors, Impact of technology on successful ageing. (pp. 188- 202). New York: Springer Publishing Company, 2003

[2] American Association of Retired Persons (AARP). Housing preferences of older adults. Washington, DC: AARP, 2003.

[3] American Society of Interior Designers. Ageing in place: Ageing and the impact of interior design. [Brochure]. Washington, DC: American Society of Interior Designers, 2003.

[4] K.R. Grant, C. Amaratunga, P. Armstrong., M. Boscoe, A. Pederson \& K. Willson.. Care for/Caring about: Women, home care and unpaid caregiving. Aurora: Ontario: Garamond Press, 2004.

[5] S.H. Zarit K.E. Reever, \& J. Bach-Peterson. Relatives of the impaired elderly: Correlates of feelings of burden. Gerontologist, 20(6), 649-655, 1980.

[6] E. Dishman. Inventing wellness systems for ageing in place. Computer, 4, 34-41, May 2004. (doi:10.1109/MC.2004.1297237)

[7] E. Dishman. Designing for the new old: Asking, observing, and performing future elders. In B. Laurel (Ed.), Design research: Methods and perspectives (pp. 41-48). Cambridge, MA: MIT Press, 2003.

[8] E. Dishman., J. Matthews, \& J. Dunbar-Jacob. Everyday health: Technology for adaptive ageing. In R.W. Pew \& S.B. VanHemel (Eds.). Technology for adaptive ageing (pp. 179-208). Washington, DC: That National Academies Press, 2003.

[9] M. Himanen. The Smart Home for Keeping Up the Life Style of the Old Ages. In: The proceedings of the ICADI, International Conference on Ageing, Disability and Independence Advancing Technology and Services to Promote Quality of Life. Track of High Technology. Dec 4-6. 2003. Washington, DC: University of Florida, American Society on Ageing, European Commission, 2003.

[10] A. Bowes.. Evaluation of Home Comforts smart home technologies initiative. Final report to South Ayrshire Council. University of Stirling, Scotland, 2003. 
[11] K. Dought.. Sheltered Housing \& Care Homes Into the 21st Century - the Role of Technology. Technology in Healthcare, 2003.

[12] J.L. Fozard, J. Rietsema, H. Bouma, \& J.A.M Graafmans. Gerontechnology: Creating enabling environments for the challenges and opportunities of ageing. Educational Gerontology, 26(4), 331-344, 2000. (doi:10.1080/036012700407820)

[13] H.W. Wahl. Research on living arrangements in old age for what? In K. W.Schaie, H.W. Wahl, H. Mollenkopf, \& F. Oswald (Eds.), Ageing independently. NewYork: Springer, 2003.

[14] I. Korhonen, J. Parkka, and M. Van Gils, "Health monitoring in the home of the future," IEEE Engineering in Medicine and Biology Magazine, vol. 23, no. 3, pp. 66-73, May/June 2003. (doi:10.1109/MEMB.2003.1213628)

[15] D.L. Hudson and M.E. Cohen, "The role of information technology in disease management," in Proc. 4th Annual IEEE Conference on Information Technology Applications in Biomedicine, p. 169-172, 2003.

[16] P.M. Orr, M.A. McGinnis, L.R. Hudson, S.S. Coberley, A. Crawford, J.L. Clarke, and N.I. Goldfarb, "A focused telephonic nursing intervention delivers improved adherence to A1c testing," Disease Management, vol. 9, no. 5, pp. 277-283, 2006. (doi:10.1089/ dis.2006.9.277)
[17] D.L. Hudson, G. Brent Hamar, P.M. Orr, J.H. Johnson, A. Neftzger, R.S. Chung, M.L. Williams, W.M. Gandy, A. Crawford, J.L. Clarke, and N.I. Goldfarb, "Remote physiological monitoring: clinical, financial, and behavioral outcomes in a heart failure population, Disease Management, vol. 8, no. 6, pp. 372-381, 2005. (doi:10.1089/dis.2005.8.372)

[18] R.W. Pew and S.B. Van Hemel, Eds., Technology for adaptive ageing, Steering Committee for the Workshop on Technology for Adaptive Ageing, National Research Council, 2004

[19] Center for Ageing Services Technologies (CAST).. The State of Technology for Ageing Services. Washington, DC: Author, 2008.

\section{AUTHORS}

S. Lam (sancheslam@gmail.com) was with New College, Oxford. He is now with the Social Sciences Division, Oxford University, UK.

W. Chung (winnie0903@hotmail.com) is with the Social Work Department, Hospital Authority, Hong Kong

Submitted December, 28, 2009. Published as resubmitted by the authors January, 20, 2010. 